\title{
Social cognition in the first year
}

\section{Tricia Striano ${ }^{1,2}$ and Vincent M. Reid ${ }^{2}$}

\author{
${ }^{1}$ Department of Pediatrics and Kennedy Center for Human Development, Vanderbilt University, Nashville, TN 37203, USA \\ ${ }^{2}$ Neurocognition and Development Group, Center for Advanced Studies, University of Leipzig, Otto-Schill Strasse 1, 04109 Leipzig, \\ Germany and Max Planck Institute for Human Cognitive and Brain Sciences, Stephanstrasse 1a, 04103 Leipzig, Germany
}

\begin{abstract}
Although the study of infancy has answered many important questions about the human capacity for social cognition, the relatively young field of developmental social cognition is far from reaching its adulthood. With the merging of developmental, behavioral and neurocognitive sciences, some growing pains are in store. New work demonstrates that research into early social cognitive development must integrate various research fields and methods in order to achieve a more robust understanding of the nature and parameters of human social cognition.
\end{abstract}

\section{Overview}

Social cognition refers to the ability to understand other people. The study of social cognition is inherently interdisciplinary. It includes everything from understanding the foundational perceptual skills that enable us to discriminate between people and objects, the complex interplay of social cues such as eye contact, body movements, tone of voice and facial expressions that help us to interpret the behavior of others, to the capacity and function of symbolic communication. Formerly, the problem of social cognition was left to social psychologists, neuroscientists, anthropologists and psycholinguists who tackled these questions among adults. However, recently, many developmental psychologists have started to address these questions. There is no better way to address a complex problem, such as that of understanding human social cognition, than to unravel its ontogeny. Here, we outline key aspects of early social cognition and describe their function and developmental trajectory. Starting with newborn infants and their predisposition to specific social information, we outline new research on how infants interact with others. We show that to best understand early social cognition, multiple methods must be used. Recent research in the field of early social cognition shows that the relationship between social cognitive skills and later abilities are more complex than was previously realized.

\section{Dyadic (person-person) interaction}

In the early months, infants primarily engage in dyadic, face-to-face interactions. These interactions are characterized by reciprocation of affect and emotions between social partners (Figure 1; dyadic). There are major changes at 2-3 months in the way that infants interact with others [1]. By the second month, infants begin to focus on the eyes and

Corresponding author: Striano, T. (striano@cbs.mpg.de)

Available online 30 August 2006. mouth of other people [2,3], and become distressed when others pose a 'still-face' and stop interacting with them [4]. Despite these transitions, newborn infants are far from asocial. Even hours after birth, infants have been found to look for longer at a face with a direct, compared with averted, gaze [5,6], an ability that will have an impact on the ability to interact with others, follow their gaze and learn about the world. Newborns are sensitive to faces, voices and eye contact [7] but do not appear to have particular social expectations or show reduced attention or affect towards a social partner who suddenly stops interacting. However, with only six weeks of interactive experience, infants show a classic still-face effect. $[8,9]$. They reduce their smiling and gazing and then attempt to re-engage the social partner. They distinguish between an adult who interacts in a relevant way by providing contingent feedback, such as smiles and vocalizations, compared with someone who interacts in an irregular way, with delayed social feedback $[4,10]$.

Within the first months, infants have had enough regular social experience to detect small perturbations - even a 1 second temporal delay - in the flow of interpersonal interaction [11-13]. Together with a sensitivity to social cues, such as eye-to-eye contact and vocal cues, by 3 months, infants have the skills to understand the relevance of the social signals necessary for learning and communication [14] (Box 1). However, many questions still remain about the early foundation of social cognition.

Given the limited attentional state of newborns, answering such questions is easier said than done, and makes it important to design optimal experimental situations in which infants can respond. Null results do not mean that a behavior or capacity for understanding does not exist. However, we might be more willing to conclude that younger infants have fewer capacities than do older ones, rather than questioning the sensitivity of the methods used or speculating on the mechanisms that might give rise to more mature social cognition. Identifying the mechanisms of change should be among the major tasks for developmental scientists. An example can be found with the recently addressed question: do six-month-olds show evidence of differentiating the intentional from the accidental actions of an adult? Behne et al. [15] addressed this topic by comparing the behavioral responses of six-, nineand 18-month-old infants' towards an adult who played with them. In some cases, the adult was suddenly unwilling to give the infant a toy (i.e. teasing); in other cases, the adult was unable to give a toy (i.e. accidentally dropping). The authors measured how much infants looked away from 


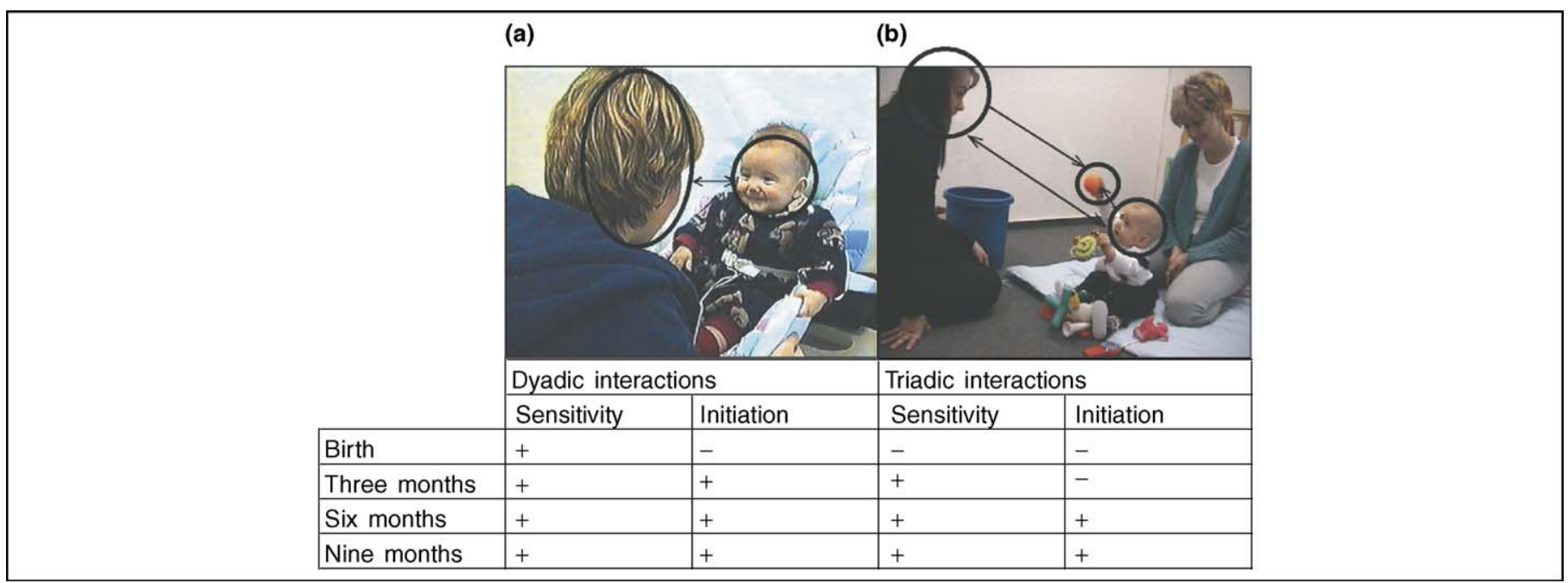

Figure 1. Developmental progression of sensitivity to dyadic and triadic relationships compared with the initiation of dyadic and triadic social interactions. (a) Dyadic

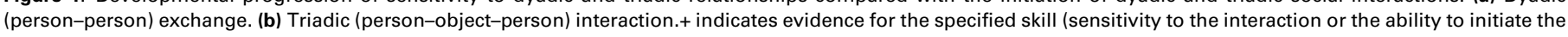
interaction) and the listed age; - indicates no evidence for the skill at the listed age.

\section{Box 1. Detecting and identifying the parameters of others}

The investigation of biological motion is important for social cognition. Behavioral research with infants suggests an early capacity to detect and interpret biological motion. This is shown by a preference to attend to biological motion when compared with other moving stimuli [42]. This research is typically conducted with pointlight displays (PLDs), depicted by points of light moving as if attached to the major joints of a moving person. PLDs are often used because a minimum amount of information is provided on what is represented by the dots.

Research into infant perception of biomechanical motion suggests that even three-month-old infants are able to discriminate some aspects of biological motion from other forms of motion [42]. Recent research has focused on neural mechanisms associated with the perception of biological motion. These studies have found that eight-month-old infants appear to process biological motion in the same manner as do adults, when compared with scrambled motion [43]. In another study, upright and inverted biological motion stimuli were presented to infants [44]. The ERP indicated a right parietal positivity for the upright but not the inverted PLDs. Even though the morphology of the infant ERP is ontogenetically unique, the topographical distribution and the latency suggest that infant and adult perception of biological motion are similar at the cognitive level $[45,46]$.

Advocates of PLDs argue that little information is provided in terms of the underlying schematics of the represented object or individual [47]. A central problem is that to perceive that certain PLD movements are 'biological', some underlying parameters of the depicted organism must be understood. However, it is argued that infant human body knowledge is poor at best. For example, only 18-month-old infants dishabituate when presented with simple corruptions to body schema $[48,49]$. Such disruptions include removing arms and legs and placing the limbs in the alternate limb location (Figure I). When adults observe the same images, the recognition of schematic disruption is virtually instantaneous. Thus, the conflict between the detection of biological motion and the corruption of body schema might be the result of the task indexing different aspects of the observed agent, potentially explaining why ERP studies suggest that infants might encode the structure of the human body [50]. Further research might resolve these issues in early social cognition.
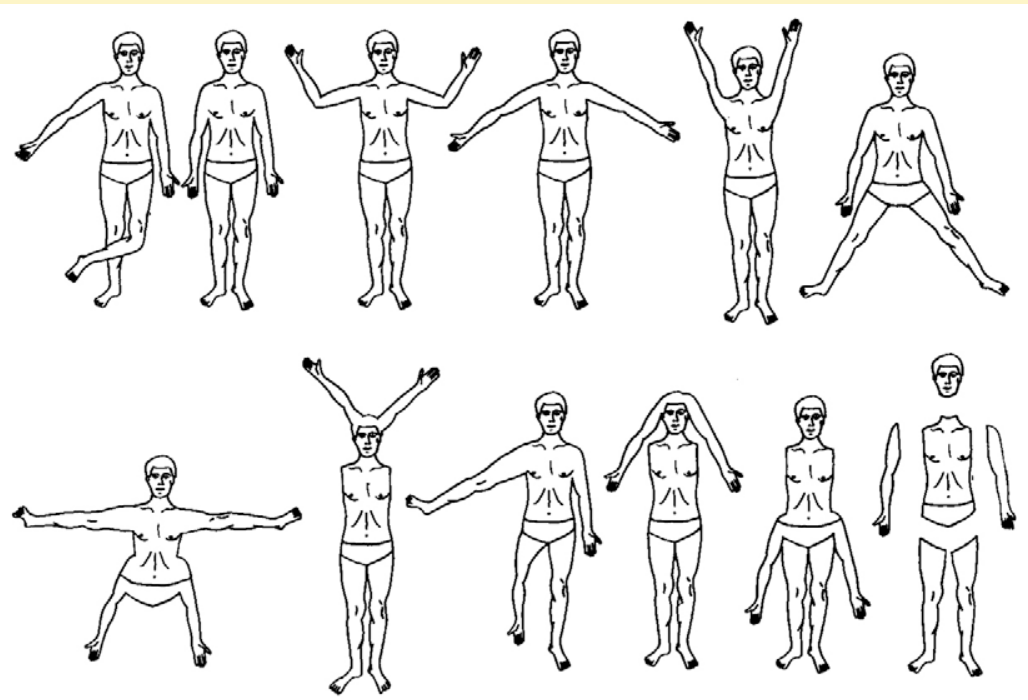

Figure I. Schematically possible (top row) and impossible (bottom row) images of the human body. Infants younger than 18 months fail to discriminate between these two conditions, suggesting a lack of understanding with respect to the normal configuration of the human body. Reproduced, with permission, from Ref. [48]. 
the adult and reached for the object. The results showed that six-month-old infants did not differentiate between an adult who was unwilling or unable to give them a toy suggesting that they could not distinguish the intentional from the accidental actions of others. However, the amount of time that infants looked at the adult, and the way that infants communicated toward the adult (such as by looking at the adult while vocalizing positively or negatively) might be a better measure of the competencies of infants. Furthermore, how do we explain that adult chimpanzees distinguish between unwilling and unable actions using a similar paradigm [16]? If it is the case that chimpanzees and nine-month-old human infants can understand intentional action but that six-month-old human infants cannot, what is the underlying mechanism that leads to this development? Here, it could be useful to employ other methods and measures (Box 2). For instance, when we measure the amount of time that a seven-month-old looks at an adult's face as a function of being teased playfully or having a toy taken away, we find evidence

\section{Box 2. Methods and techniques in infancy research}

Techniques to assess infant perceptual and cognitive abilities can be divided into two distinct groups. Behavioral paradigms assess overt infant behavior in response to changes in the environment. The assessment of infant brain function can determine infant cognitive processes in the absence of an overt behavioral response. Each technique has distinct advantages and disadvantages, as outlined below.

\section{Common behavioral paradigms}

Preferential looking: Two objects or events are presented to infants at the same time. Measures of infant looking time are made and inferences can be made on whether infants discriminate between the stimuli.

Habituation studies: A picture or stimulus is presented to infants many times. A new stimulus is then presented, and looking times are measured to assess discrimination between the previous stimulus and the new stimulus. If infants spend longer looking at the new stimulus, this indicates that they detect differences between the old and the new stimulus [51].

\section{Common techniques in cognitive neuroscience}

Electrophysiological: These measures, such as event-related potentials, assess electrical brain activity associated with the presentation of stimuli. Typically, the infant repeatedly observes the same stimulus and comparisons are made between brain activity before the stimuli and during the presentation of the stimuli. This technique provides millisecond to millisecond information on how the infant brain processes stimuli; however, it lacks specific information on the location of detected neural activity. Importantly, electrophysiological techniques do not require overt behavioral responses and therefore permit the investigation of social cognitive processes that might be difficult to investigate with behavioral techniques alone [52].

Hemodynamic: The use of functional magnetic resonance imaging has not been common with early developmental populations. Specifically, little is known regarding the effects of high magnetic fields on the developing brain. Additionally, infants must remain motionless, or data cannot be obtained. However, a new method of investigating the functional brain has been developed. Near-infrared spectroscopy can measure changes in cerebral oxygenation in human infants [53]. This technique involves emitting an infrared light through the infant's skull, to be received by a detecting array. Blood oxygen levels can then be calculated. Near-infrared spectroscopy holds much promise for mapping structural and functional brain relationships in the developing infant brain. of action discrimination [17]. Research is needed to establish the behavioral and neural correlates of social cognitive understanding as a function of development and species. If different neural correlates could be established between six- and nine-month olds and chimpanzees, we would be in a much better position to make claims about both the ontogeny and phylogeny of social cognition.

\section{From dyadic to triadic (person-object-person) relations}

The interpretation of dyadic behavioral cues present in the way that other people move, talk and relate is probably a predominant means by which we infer the mental states of others [18-20]. A sensitivity to dyadic cues might also be crucial in the identification of infants with communicative impairments, such as autism (Box 3).

A key transition in early development is from participating in face-to-face (dyadic) interactions to engaging in person-object-person (triadic) interactions. Triadic interactions involve two people in relation to some third external object, situation or event. These interactions are essential for the development of abilities such as language and imitation $[21,22]$. For instance, as shown in Figure 1 (triadic), to learn the name for a novel object, the infant must be able to detect when the interactive partner is communicating relevant information directed at the self, and to what this information refers.

Because of the inclination for dyadic face-to-face interaction in the early months, it has been assumed that young

\section{Box 3. Social sensitivities in autism}

Understanding the development of social interaction should provide a tool for the identification of infants at risk for communicative impairments, such as autism. Children with autism attend to perfect (nonsocial) compared with imperfect (social) contingencies. [54]. Understanding the sensitivity of infants to social contingencies and what accounts for transitions might be key in predicting social cognitive deficits. A first step in successful communication is understanding when a social signal is directed at and intended for the self. In adulthood, there are specialized brain regions activated when a social signal conveys communicative intention. When adults hear their name or are the visual target of someone's direct gaze, the paracingulate cortex and temporal poles activate [55]. Regardless of modality (auditory or visual), the adult brain is ready to detect another's intention to communicate. Among the core deficits in autism is an inability to detect people, to orientate towards one's name [56] or to detect relevant social cues [57]. The capacity to detect the relevance of social and emotional cues relies upon a sensitivity to other people's behavior and an understanding of the relationship between self and other [58].

Alongside a range of social and cognitive impairments - ranging from a dysfunctional attention system to avoidance of social signals [59-62] - a disrupted mirror system probably contributes to the inability to comprehend the correspondence between self and other, and will have cascading effects for later social cognitive skills observed in autism [58]. Although the development of the mirror neuron system in human ontogeny is unknown, by birth, infants can imitate the actions of others [63]. This suggests an awareness of the correspondence between self and other. With such skills in place, infants can begin to learn about and use the social cues that others provide. Typically, developing infants are highly selective in determining from whom and under what circumstances to use information that others provide $[64,65]$. Research showing activation of adult brain systems when information is directed at the self fits well with research showing the effect of communicative facial and vocal cues on infant behavior. [66-68]. 
infants are simply not capable of engaging in triadic interactions. Based on studies that considered infants' capacity for joint attention only at nine months of age and later [23,24], it has been concluded that the capacity for joint attention emerges at nine months of age or later. Recent research with infants under nine months of age tells a different story. By five months, infants actively coordinate visual attention between people and objects $[25,26]$. Although it is clear that there are developmental transitions toward the end of the first year, the basis of these developments is simply not understood.

\section{Joint attention: so what's the early function?}

Only recently has the infant's capacity for triadic interaction been investigated. In one recent study, three-, six- and nine-month-old infants interacted with an adult and objects. The infants and the adult interacted in a dyadic way, and then different types of triadic interactions were introduced. By three months of age, infants discriminated among various triadic interactions [27]. But why should infants be sensitive to triadic attention if it is not functional? Some might conclude that a three-month-old infant cannot use the visual attention of others to learn language, new actions or about the world. The fact of the matter is that these issues have not been addressed. There is some evidence to suggest that a sensitivity to triadic attention, even in three- to four-month-old infants, is functional [28]. This makes sense because research shows links between the capacity for triadic attention (i.e. gaze following) at ten months and language capacities at 18 months $[22,29]$. However, we need to know much more about how social sensitivities in early development might relate to, and predict, later cognitive functioning. What we do know is that even four-month-old infants are attuned to the eye gaze of others and use eye gaze cueing in processing objects. In one study [30], four-monthold infants watched a video presentation of an adult gazing towards one of two objects. When presented with the same objects a second time, infants gazed towards the uncued object for a significantly longer period of time - suggesting that it was more novel. This means that four-month-old infants not only followed the gaze of the adult, but also acquired information about the object.

Beyond sensitivity to eye-gaze cueing, triadic cues assist infants in object processing. In general, joint attention helps young infants to establish the relevance of social information. Cues such as eye contact [31] and tone of voice [32] help infants to establish when information is intended for them. They can then use these cues to guide their attention towards the world and to learn more effectively. Evidence from the brain activity of infants supports this view. Reid et al. [33] investigated object processing among four-month-old infants in an event-related potential (ERP) study. Infants viewed an adult's face onscreen, and the eyes of the adult gazed at an object. In the test trials, infants viewed the objects for a second time. Infants rapidly exhibited enhanced neural processing (indexed by a positive slow wave) of the uncued object during test trials. Thus, the cued object was processed and more familiar to the infant in the test trials than was the uncued object. This study reveals both a sensitivity to triadic cues and their functional use by four months of age.
One of the challenges facing infancy researchers is how to design effective paradigms that best reveal what developing infants can do and what they might be capable of processing. To determine what infants understand, it is important to make a distinction between the infants' sensitivity to social information and their capacity to initiate or produce social communication. Considering these two domains separately might contribute uniquely to our understanding of human social cognition (Figure 1). For instance, take the problem of assessing the neural correlates of social cognition using computer-displayed images, and when presenting people as stimuli over television. It is known that children do not treat television images as live events [34,35] and that the infant brain processes two-dimensional and threedimensional stimuli differently [36]. These findings are consistent with research showing that mirror neurons in monkeys fail to fire when observed actions are presented via television [37]. To overcome the problems associated with unnatural social events, it is necessary to design studies that optimize the attention of infants and keep social interaction as natural as possible. In this regard, the development of the first live-interactive paradigms to study how the infant brain processes information has proven successful [38]. However, far more research is required to understand how stimulus materials interact with the development of social cognitive skills.

\section{Interactive ERP paradigms}

In one recent study, the neural correlates of triadic attention were assessed in nine-month-old infants [38]. Infants faced a computer monitor. An adult sat across from the

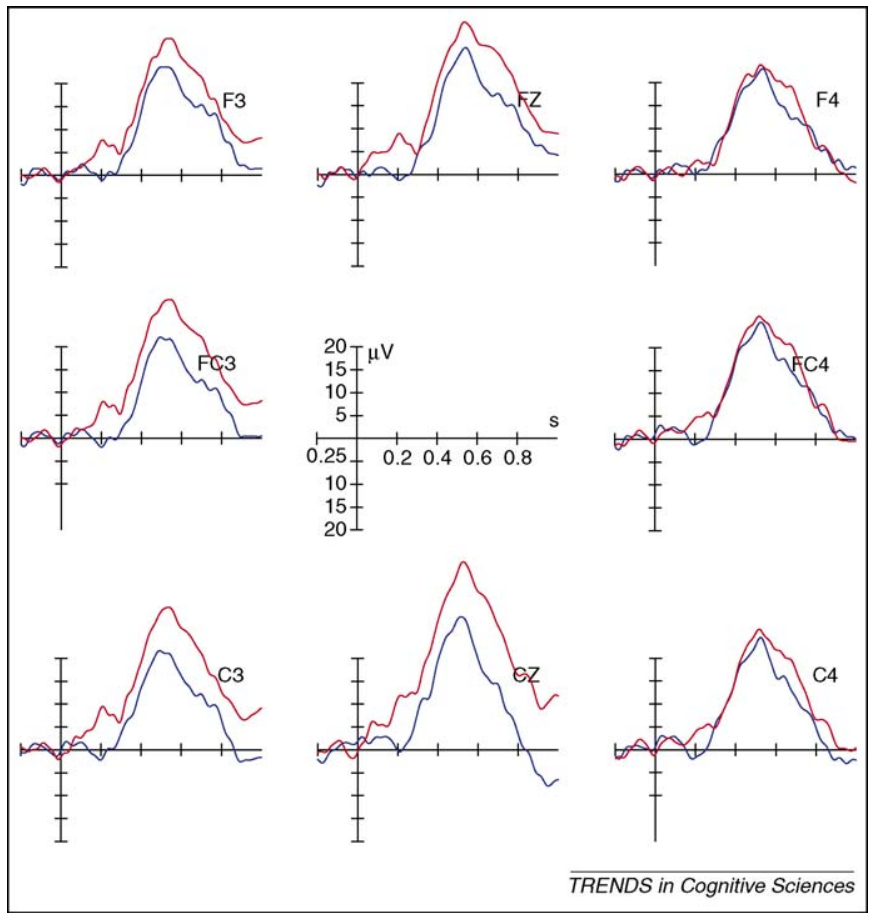

Figure 2. Event-related potential responses to observing an object in a joint attention (JA) condition (gray) and a nonjoint attention condition (black). The joint attention condition produces a larger amplitude negative component when compared with the no joint attention condition, peaking at $400 \mathrm{~ms}$ after stimulus onset. This suggests a greater allocation of attentional resources by the infant during the joint attention interaction. The labels indicate the electrode site in the international 10-20 EEG system. ' $\mathrm{F}$ ' channels are frontal and ' $\mathrm{C}$ ' channels are central scalp sites. Reproduced, with permission, from Ref. [31]. 


\section{Box 4. Questions for future research}

- How do infants learn about the relevance of social signals?

- What brain mechanisms are involved in the detection of relevant social signals, and how does this process work in typically developing infants and those with social cognitive impairments?

- What is the role of eye gaze and other social cues in early learning?

- What are the universal social cues to which infants are sensitive?

- What are the main components of dyadic interaction, such as eye gaze, vocalizations, head orientation and physical contact, and what is their order of importance for social cognition as a function of development?

- During triadic interaction, what initial information is important for understanding referential looking between a person, an object and the self?

- How do infants at risk for autism process discrete aspects of dyadic and triadic interactions, such as eye gaze cueing or vocalizations?

- When does the biological motion detection system mature?

- What is the relationship between detecting biological motion and detecting body schema?

infants and interacted in various ways. In a joint attention context, the adult gazed at the infant's face and then to a novel object that was displayed on a computer screen for 1 second. In the nonjoint attention context, the adult gazed only at the novel object. Within a period of 5 seconds, and for each trial included in analysis, infants looked at the adult's face and then to the novel object on the screen. The electrical brain activity of the infants was then measured as they viewed the novel objects presented on the computer screen.

The infant brain processed objects differently depending on social context. As shown in Figure 2, the negative component of the infant event-related potential, a neural correlate indexing attentional processes [39], was enhanced in amplitude (red line) when infants were engaged in a joint attention interaction compared with a nonjoint attention interaction. Given the interplay between joint attention and later cognitive skills [40,41], the results suggest a general learning mechanism that might underlie a range of cognitive developments. To ascertain whether this is the case, such paradigms should be applied to a range of cognitive skills. Whatever the outcome, the application of ERP techniques to social situations stands to greatly inform the wider developmental sciences and help to resolve the many questions that remain on early social cognition (Box 4).

\section{Summary}

We have outlined recent advances in our understanding of early social cognition. It has been found that young infants have many of the rudimentary skills needed for more mature aspects of social cognition. Through the study of early development, it is possible to understand what types of experiences and maturations are necessary to learn from and relate to others, including predicting and interpreting the behavior of others. Many social cognitive milestones have been established but we need now to understand the mechanisms of developmental change and how these account for brain and behavioral functioning at different ages. Understanding the mechanisms of development is an interdisciplinary problem that demands the study of infancy and the integration of fields such as neuroscience, social psychology and anthropology. Finally, it is only through the use of multiple methods that new insights into the development of the infant mind will be found.

\section{Acknowledgements}

The authors are supported by the Sofja Kovalevskaja Award, granted by the Alexander von Humboldt Foundation, donated by the Federal Ministry of Education and Research, to T.S.

\section{References}

1 Lavelli, M. and Fogel, A. (2005) Developmental changes in mother-infant face-to-face communication: birth to 3 months. Dev. Psychol. 38, 288-305

2 Johnson, M.H. and Morton, J. (1991) Biology and Cognitive Development: The Case of Face Recognition. Blackwell

3 Jaffe, J. et al. (2001). Rhythms of dialogue in infancy: coordinated timing and social development. Monograph of the Society for Research in Child Development 265, 66, Washington, DC

4 Striano, T. et al. (2005) Sensitivity to social contingencies between 1 and 3 months of age. Dev. Sci. 8, 509-519

5 Farroni, T. et al. (2002) Eye contact detection at birth. Proc. Natl. Acad. Sci. U. S. A. 99, 9602-9605

6 Macchi Cassia, V. et al. (2001) Face preference at birth: the role of an orienting mechanism. Dev. Sci. 4, 101-108

7 Rochat, P. and Striano, T. (1999) Social cognitive development in the first year. In Early Social Cognition (Rochat, P., ed.), pp. 3-34, Lawrence Erlbaum Associates

8 Adamson, L.B. and Frick, J.E. (2003) The still-face: a history of a shared experimental paradigm. Infancy 4, 451-473

9 Striano, T. and Bertin, E. (2005) Coordinated affect with mothers and strangers: a longitudinal study of joint attention between 5 and 9 months of age. Cogn. Emot. 19, 781-890

10 Nadel, J. et al. (1999) Expectancies for social contingency in 2-month-olds. Dev. Sci. 2, 164-173

11 Striano, T. et al. (2006) Sensitivity to interpersonal timing at 3 and 6 months of age. Interaction Studies 7, 251-271

12 Nadel, J. et al. (1999) Expectancies for social contingency in 2-month-olds. Dev. Sci. 2, 164-173

13 Keller, H. et al. (1999) Temporal contingency as an independent component of parenting behavior. Child Dev. 70, 474-485

14 Striano, T. and Bertin, E. (2004) Contribution of facial and vocal cues in the still-face by 4 month old infants. Infant Behav. Dev. 27, 499-508

15 Behne, T. et al. (2005) Unwilling versus unable: infants' understanding of intentional action. Dev. Psychol. 41, 328-337

16 Call, J. et al. (2004) Unwilling versus unable: chimpanzees understanding of human intentional action. Dev. Sci. 7, 488-498

17 Striano, T. and Vaish, A. Seven- to 9-month-old infants use facial expressions to interpret otherś action. Br. J. Dev. Psychol. DOI:10.1348/026151005X70319

18 Iacoboni, M. et al. (2005) Grasping the intentions of others with one's own mirror neuron system. PLoS Biol. 3, e79

19 Hadjikhani, N. and de Gelder, B. (2003) Seeing fearful body expressions activates the fusiform cortex and amygdala. Curr. Biol. 13, 2201-2205

20 de Gelder, B. (2005) Nonconscious emotions: new findings and perspectives on nonconscious facial expression recognition and its voice and whole body contexts. In Emotion and Consciousness (Feldman Barrett, L. et al., eds), pp. 123-149, The Guilford Press

21 Baldwin, D.A. and Moses, L.J. (2001) Links between social understanding and early word learning: challenges to current accounts. Soc. Dev. 10, 309-329

22 Brooks, R. and Meltzoff, A.N. (2005) The development of gaze following and its relation to language. Dev. Sci. 8, 535-543

23 Carpenter, M. et al. (1998) Social cognition, joint attention, and communicative competence from 9 to 15 months of age. Monogr. Soc. Res. Child Dev. 63 (4, Serial No. 255), 1-143

24 Tomasello, M. et al. (2005) Understanding and sharing intentions: the origins of cultural cognition. Behav. Brain Sci. 28, 675-691

25 Striano, T. and Bertin, E. (2005) Relation among joint attention skills in 5- to 10-month-old infants. Br. J. Dev. Psychol. 23, 1-11

26 Flom, R. and Pick, A.D. (2005) Experimenter affective expression and gaze following in 7-month-olds. Infancy 7, 207-218 
27 Striano, T. and Stahl, D. (2005) Sensitivity to triadic attention in early infancy. Dev. Sci. 4, 333-343

28 Hood, B.M. et al. (1998) Adult's eyes trigger shifts of visual attention in human infants. Psychol. Sci. 9, 53-56

29 Baldwin, D.A. and Moses, L.J. (2001) Links between social understanding and early word learning: challenges to current accounts. Soc. Dev. 10, 309-329

30 Reid, V.M. and Striano, T. (2005) Adult gaze influences infant attention and object processing implications for cognitive neuroscience. Eur. J. Neurosci. 21, 1763-1766

31 Farroni, T. et al. (2004) Mechanisms of eye gaze perception during infancy. J. Cogn. Neurosci. 16, 1320-1326

32 Grossmann, T.et al. (2006) Crossmodal binding of emotional information from face and voice in the infant brain. Dev. Sci. 9, 309-315

33 Reid, V.M. et al. (2004) Eye gaze cuing facilitates neural processing of objects in 4 month old infants. Neuroreport 15, 2553-2556

34 Troseth, G.L. (2003) Getting a clear picture: young children's understanding of a televised image. Dev. Sci. 6, 247-253

35 Barr, R. and Hayne, H. (1999) Developmental changes in imitation from television during infancy. Child Dev. 70, 1067-1081

36 Carver, L.J. et al. (2006) Event-related potential (ERP) indices of infants' recognition of familiar and unfamiliar objects in two and three dimensions. Dev. Sci. 9, 51-62

37 Ferrari, P.F. et al. (2003) Mirror neurons responding to the observation of ingestive and communicative mouth actions in the monkey ventral premotor cortex. Eur. J. Neurosci. 17, 1703-1714

38 Striano, T. et al. (2006) Neural mechanisms of joint attention in infancy. Eur. J. Neurosci. 23, 2819-2823

39 Richards, J.E. (2003) Attention affects the recognition of briefly presented visual stimuli in infants: an ERP study. Dev. Sci. 6, 312-328

40 Leekam, S.R. and Ramsden, C.A. (2006) Dyadic orienting and joint attention in preschool children with autism. J. Autism Dev. Disord. 36, 185-197

41 Markus, J. et al. (2000) Individual differences in infant skills as predictors of child-caregiver joint attention and language. Soc. Dev. 9, 302-315

42 Bertenthal, B.I. (1993) Infants' perception of biomechanical motions: intrinsic image and knowledge-based constraints. In Visual Perception and Cognition in Infancy (Granrud, C., ed.), pp. 175-214, Erlbaum

43 Hirai, M. and Hiraki, K. (2005) An event-related potentials study of biological motion perception in human infants. Brain Res. Cogn. Brain Res. 22, 301-304

44 Reid, V.M. et al. (2006) The perception of biological motion by infants: an event-related potential study. Neurosci. Lett. 395, 211-214

45 Jokisch, D. et al. (2005) Structural encoding and recognition of biological motion: evidence from event-related potentials and source analysis. Behav. Brain Res. 157, 195-204

46 Wheaton, K.J. et al. (2001) Neuronal responses elicited to viewing the actions of others. Vis. Neurosci. 18, 401-406

47 Golinkoff, R. et al. (2002) Young children can extend motion verb labels to point-light displays. Dev. Psychol. 38, 604-615

48 Slaughter, V. and Heron, M. (2004) Origins and early development of human body knowledge. Monogr. Soc. Res. Child Dev. 69, 103-113
49 Slaughter, V. et al. (2002) Development of preferences for the human body shape in infancy. Cognition 85, B71-B81

50 Gliga, T. and Dehaene-Lambertz, G. (2005) Structural encoding of body and face in human infants and adults. J. Cogn. Neurosci. 17, 1328-1340

51 Sirois, S. and Mareschal, D. (2002) Models of infant habituation. Trends Cogn. Sci. 6, 293-298

52 Johnson, M.H. et al. (2001) Recording and analyzing high density ERPs with infants using the Geodesic Sensor Net. Dev. Neuropsychol. 19, 295-323

53 Csibra, G. et al. (2004) Near infrared spectroscopy reveals neural activation during face perception in infants and adults. J. Pediatr. Neurol. 2, 85-89

54 Gergely, G. et al. (2002) Contingency perception and the role of contingent parental reactivity in early socio-emotional development: some implications for developmental psychopathology. In Imitation, Action et Intentionnalité (Nadel, J. and Decety, J., eds), pp. 113-132, Press Universitaires de France

55 Kampe, K.K. et al. (2001) Reward value of attractiveness and gaze. Nature 413, 589

56 Osterling, J. and Dawson, G. (1994) Early recognition of children with autism: a study of first birthday home video tapes. J. Autism Dev. Disord. 24, 247-257

57 Klin, A. et al. (2002) Defining and quantifying the social phenotype in autism. Am. J. Psychiatry 159, 895-908

58 Dapretto, M. et al. (2006) Understanding emotions in others: mirror neuron dysfunction in children with autism spectrum disorder. Nat. Neurosci. 9, 28-30

59 Baron-Cohen, S. (2005) Testing the extreme male brain (EMB) theory of autism: let the data speak for themselves. Cogn. Neuropsychiatry 10, $77-81$

60 Dawson, G. et al. (2004) Defining the early social attention impairments in autism: social orienting, joint attention, and responses to emotions. Dev. Psychol. 40, 271-283

61 Hobson, R.P. and Lee, A. (1999) Imitation and identification in autism J. Child Psychol. Psychiatry 40, 649-659

62 Volkmar, F.R. et al. (2004) Autism and pervasive developmental disorders. J. Child Psychol. Psychiatry 45, 135-170

63 Meltzoff, A.N. and Prinz, W., (eds) (2002) The Imitative Mind: Development, Evolution, and Brain Bases. Cambridge University Press

64 Striano, T. et al. (2006) Selective looking by 12 -month-olds to a Temporally Contingent Partner. Interact. Stud. 7, 233-250

65 Striano, T. et al. Eye contact influences neural processing of emotional expressions in 4-month-old infants. Soc. Cogn. Affect. Neurosci. DOI:10.1093/scan/ns1008

66 Mumme, D.L. et al. (1996) Infants' responses to facial and vocal emotional signals in a social referencing paradigm. Child Dev. 67, 3219-3237

67 Vaish, A. and Striano, T. (2004) Is visual reference necessary? Vocal versus facial cues in social referencing. Dev. Sci. 7, 261-269

68 Striano, T. et al. The meaning of infants' looks: information gathering and comfort seeking? Br. J. Dev. Psychol. DOI:10.1348/ $026151005 \times 67566$

\section{Elsevier joins major health information initiative}

Elsevier has joined with scientific publishers and leading voluntary health organizations to create patientINFORM, a groundbreaking initiative to help patients and caregivers close a crucial information gap. patientINFORM is a free online service dedicated to disseminating medical research.

Elsevier provides voluntary health organizations with increased online access to our peer-reviewed biomedical journals immediately upon publication, together with content from back issues. The voluntary health organizations integrate the information into materials for patients and link to the full text of selected research articles on their websites.

patientINFORM has been created to enable patients seeking the latest information about treatment options online access to the most up-to-date, reliable research available for specific diseases.

For more information, visit www.patientinform.org 